Original Article

\title{
Ergometer cycling improves the ambulatory function and cardiovascular fitness of stroke patients - a randomized controlled trial
}

\author{
Ernest Kwesi Ofori, PT, MSc ${ }^{1)}$, Emmanuel Frimpong, PT, Physiol, $\mathrm{PhD}^{2)^{*}}$, \\ Adeolu Ademiluyi, PT ${ }^{1)}$, Olajide Ayinla Olawale, PT, Physiol, PhD ${ }^{3)}$ \\ 1) Department of Physical Therapy, College of Applied Health Sciences, University of Illinois \\ at Chicago, USA \\ 2) Movement Physiology Research Laboratory, School of Physiology, Faculty of Health Sciences, \\ University of the Witwatersrand, Johannesburg, South Africa \\ 3) Department of Physiotherapy, Faculty of Clinical Sciences, College of Medicine, University of \\ Lagos, Nigeria
}

\begin{abstract}
Purpose] The aim of this study was to assess the effects of ergometer cycling on the ambulatory function and cardiovascular fitness of patients with stroke in the sub-acute phase. [Participants and Methods] Twenty (20) patients with stroke in the sub-acute phase were randomly allocated to either an ergometer cycling group ( $\mathrm{n}=10)$ or a control group $(\mathrm{n}=10)$. The experimental (ergometer cycling) group performed cycling exercises in addition to conventional physiotherapy for 60 minutes per session, three times per week for 8 weeks. The control group only received conventional physiotherapy for the same duration as the experimental group. Assessments of participants' functional ambulatory category, ambulatory velocity, 6-minute walk test, heart rate and blood pressure were conducted at baseline and at the end of the 8-week intervention. [Results] The means of the ambulatory velocity and distance walked in 6 minutes were significantly higher in the ergometer cycling group than those of the control group at week 8. However, the increase in the FAC score was not significant. The means of heart rate, systolic and diastolic blood pressures significantly decreased in the ergometer cycling group compared to the control group at the end of the 8-week of intervention. [Conclusion] This study demonstrated that ergometer cycling improved the ambulatory function and cardiovascular fitness of patients with stroke in the sub-acute phase.

Key words: Stroke, Ergometer cycling, Ambulatory function
\end{abstract}

(This article was submitted Jun. 22, 2016, and was accepted Sep. 14, 2016)

\section{INTRODUCTION}

Stroke is a leading cause of long-term disabilities in adults. It is characterized by hemiparesis, walking inability without assistance, and dependence on others for the performance of activities of daily living (ADL) ${ }^{1)}$. Performing continuous and smooth reciprocal movements such as ambulation (walking), is a very difficult task for hemiparetic patients with stroke ${ }^{2}$. This may be due to residual motor weakness or paresis, spasticity, poor motor control and lower limb coordination, postural imbalance and loss of sensation ${ }^{3-5}$ ). In addition to neurological impairments, walking dysfunction after stroke is also associated with the cardiovascular and musculoskeletal consequences of the disease and physical inactivity ${ }^{6}$.

Musculoskeletal deficits (including muscle atrophy and contractures) and cardiovascular problems (such as decreased maximal oxygen consumption) as well as low aerobic endurance significantly affect stroke patients' ADL performance and in severe cases, may lead to death ${ }^{7)}$. Thus, the physical deconditioning along with age-associated declines in fitness and

*Corresponding author. Emmanuel Frimpong (E-mail: frimemma@gmail.com)

(C2019 The Society of Physical Therapy Science. Published by IPEC Inc.

This is an open-access article distributed under the terms of the Creative Commons Attribution Non-Commercial No Derivatives (by-nc-nd) License. (CC-BY-NC-ND 4.0: http://creativecommons.org/licenses/by-nc-nd/4.0/) 
muscle mass can further contribute to activity intolerance, compromising patients' capacity to meet the high-energy demands of hemiparetic walking ${ }^{89}$ ). Furthermore, multiple comorbidities associated with first time and recurrent strokes including hypertension, coronary heart disease, impaired glucose tolerance, diabetes, depression and obesity worsen recovery and compound the loss in movement and overall function ${ }^{10,11)}$.

Although physical fitness is important for the performance of everyday activities, muscle strength and cardiorespiratory fitness are impaired in patients with stroke ${ }^{12}$. However, aerobic exercise reduces the cardiovascular demands that walking makes on patients with stroke ${ }^{6}$. Increased fitness levels may benefit a range of post-stroke problems by reducing fatigue, the energy cost of hemiparetic gait, and the incidence of falls and fractures, as well as improve patients' independence, mood and quality of life ${ }^{13)}$.

A major rehabilitation objective for patients with motor impairments in the lower limbs is recovery of walking capacity ${ }^{14)}$. Thus, restoring walking ability is the major objective of lower limb rehabilitation after stroke ${ }^{14)}$. However, the degree of recovery depends on several factors such as location and severity of lesion, capacity for adaptation through training, and the duration of stroke, where recovery is high in the early months after stroke ${ }^{4}$. Therefore, motor function recovery strategies after stroke must include methods directed towards recovery of walking capacity ${ }^{15)}$. Several studies have employed different exercise interventions to restore ambulatory capacity or function and cardiovascular fitness after stroke ${ }^{16-24)}$. Most of these studies used exercise training on a treadmill with or without weight-support as an intervention for improving ambulatory function and cardiovascular fitness. The outcome measures commonly used were blood pressure (BP), heart rate (HR), the six-minute walk test (6-MWT), ambulatory velocity (AV) or the ten-metre walk test (10-MWT), and the functional ambulatory category (FAC) score ${ }^{17-20,25,26)}$.

The current approach towards stroke rehabilitation emphasizes task-specific training, which involves intense practice of functional tasks ${ }^{4}$. Cycling can improve functional mobility and can act as a pseudo-walking task-oriented exercise ${ }^{27)}$. Cycling shares a similar kinematic pattern with walking because both are cyclical, require reciprocal flexion and extension movements of the hip, knee, and ankle, and both require alternating and coordinated antagonist muscle activation ${ }^{28,29)}$. Cycling can be used as a method to counter the consequences of immobility ${ }^{7)}$. This type of training improves the aerobic capacity, strength, and cardiopulmonary function of individuals ${ }^{15)}$. In addition, cycling exercise also facilitates muscle control of the lower limbs, which may allow putting more weight on the affected leg while standing ${ }^{30}$. Moreover, ergometer cycling requires less balance ability, which makes it useful for training patients with or without neurological deficits who have difficulty in maintaining balance and independent gait ${ }^{29}$. Furthermore, the ability to exercise while sitting means cycling is welltolerated by patients with stroke in the acute, sub-acute or chronic phases ${ }^{31)}$. Cycling exercise has elicited positive outcomes for walking performed by patients with chronic stroke $\left.\mathrm{s}^{7}, 32,33\right)$, and patients with incomplete spinal cord injury ${ }^{34)}$. However, few studies have evaluated the therapeutic effects of ergometer cycling on the ambulatory function and cardiovascular fitness of patients with stroke in the sub-acute phase. Moreover, studies have shown that there is greater chance of recovery during the sub-acute stage after stroke ${ }^{4,5}$. Therefore, the purpose of this study was to assess the effects of ergometer cycling on the ambulatory function and cardiovascular fitness of patients with stroke in the sub-acute phase.

\section{PARTICIPANTS AND METHODS}

The study was conducted at the Physiotherapy Department of the Korle Bu Teaching Hospital (KBTH) in Accra, Ghana. KBTH is the largest referral hospital in Ghana and has over 1,600 beds. The hospital receives referrals from all over the country and neighbouring West African countries. KBTH also serves as the teaching hospital of the College of Health Sciences (CHS) of the University of Ghana. The study population comprised patients with stroke receiving care at the hospital. The participants were hemiparetic patients with recent stroke referred to the Physiotherapy Department for rehabilitation. Patients were recruited into the study if they had: first or second stroke resulting in right or left side hemiparesis, muscle power of at least grade three, and could walk at least 10 metres with or without assistive devices. Patients were excluded from the study if they had bilateral hemiparesis, an FAC score of less than grade three, muscle power of less than grade three, fracture of the lower limb, aphasia and cognitive impairment, cardiac arrhythmias, or any condition for which exercise is contraindicated ${ }^{16)}$.

The Ethical and Protocol Review Committee (EPRC) of the University of Ghana School of Allied Health Sciences approved the study protocol. All participants gave their written informed consent after receiving explanations of the study protocol and the potential risks that could be encountered. The study was advertised via posters and verbal invitation. Eligible patients were randomly divided into two (2) groups: an experimental group or ergometer cycling group (ErCG), and a non-ergometer cycling or control group (CG). Randomization was done using cards bearing the names of the groups and the number of participants. Ten participants were allocated to each group (Fig. 1). Both groups received three sessions of treatment per week for eight weeks.

The cycling exercises began with familiarization sessions in which participants were introduced to the protocol and acquainted themselves with ergometer cycling. The EnrafNonius ergometer bicycle (EN cycle 970, Holland) was used in this study. Based on the familiarization session, the initial revolutions of pedaling and resistances were individually set for each participant. In addition, participants were instructed on what to do during the study period including: resting periods during cycling, hydration, communicating adverse symptoms, and prevention of falls.

Treatments for participants in the ErCG comprised warm-ups, cycling exercise and cool-downs. The warm-up (5 minutes) 
included overground walking and cycling at a self-selected pedaling speed. The cool-down sessions (5 minutes) involved slow pedaling, passive stretching of the lower limbs and controlled breathing exercises. Prior to the exercise, the resting heart rate (HR) and blood pressure (BP) of the participants were monitored to ensure they were clinically stable before exercise. Participants were assisted to safely mount the ergometer bicycle. The height of the seat was adjusted to ensure postural balance, upright seating and firm contact of the feet with the pedals. Participants who could not grip firmly had their hands strapped to the handle of the ergometer bicycle. The foot of the affected limb was buckled

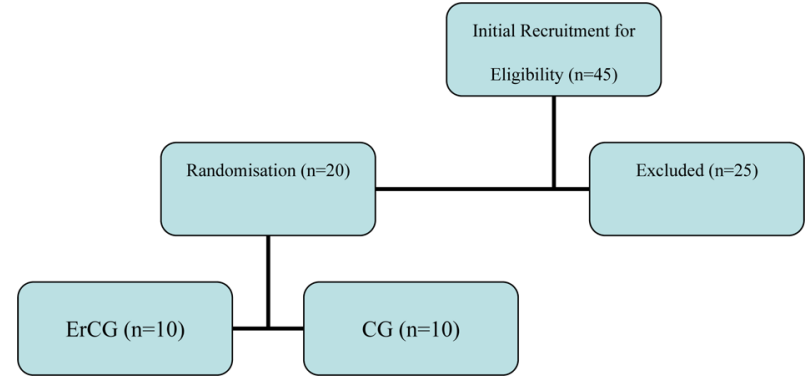

Fig. 1. Flow chart of the study design onto the pedal.

Treatment for the ErCG lasted for 60 minutes per session.

In addition to ergometer cycling, the participants also received conventional physiotherapy. The cycling was performed for 30 minutes per session, three times per week for 8 weeks. Thus, a total of 720 minutes of cycling exercises were performed over the 8-week period. The intensity of the cycling was targeted between 10 (light) and 15 (hard or heavy) on the Borg scale of rating of perceived exertion (RPE) ${ }^{35)}$. A low resistance set at 1 of 4 on the ergometer bicycle was applied to allow a cycling speed of 40 to 60 revolutions per minute (rev/min). Thus, participants performed the cycling at $40 \mathrm{rev} / \mathrm{min}$ at the beginning and $60 \mathrm{rev} / \mathrm{min}$ towards the end of the study. Participants were encouraged to increase the number of revolutions per minute throughout the study. The control group (CG) received only conventional physiotherapy comprising passive, active assisted, active and strengthening exercises as well as balance and walking re-training for the same duration as the ErCG.The ErCG and CG were assessed at baseline and at the end of the 8th week for the following outcome measures: heart rate, blood pressure, 6-minute walk test, ambulatory velocity measured by the 10-metre walk test, and functional ambulatory category (FAC) score.

Resting heart rate was measured at rest with an electronic monitor (Omron, UK). Following 10 minutes of resting in a seated position, BP was measured using an aneroid sphygmomanometer at the brachial artery with the arm supported at the level of the heart. The BP was recorded as a ratio of systolic blood pressure (SBP) mmHg to diastolic blood pressure (DBP) $\mathrm{mmHg}$.

The 6-minute walk test was performed to assess the muscular endurance and walking capacity of the participants. Participants walked around the 40-metre walkway of the therapeutic gymnasium for 6 minutes. The total distance covered during the 6 minutes was then calculated in metres. Prior to the test, participants were told that they could rest by either sitting or standing upon request and that they could walk with or without walking aids ${ }^{36}$.

The 10-MWT was conducted to assess the ambulatory velocity of the participants over a ten-metre walkway. A 14-meter walkway was marked on the floor of the gymnasium. This was done to eliminate acceleration and deceleration affecting the test. Participants were told before the test that they could walk with or without a walking aid at self-selected walking speed ${ }^{37)}$. A stop-watch was used to record the time taken by the participants to cover the ten-metre distance in the middle of the 14-metre walkway. The stop-watch was started when the participants crossed the 2-metre line, and stopped when they crossed the 12-metre line, and the time was recorded in seconds. Three trials were performed and the average time calculated ${ }^{16)}$.

The functional ambulatory category was assessed while participants were walking to evaluate the level of dependency of participants in performing functional activities. The FAC is a six-point ordinal rating scale that reflects the assistance a person requires when walking. This scale allows easy classification of patients in respect of their walking ability, and the maximum score signifies the ability to ambulate independently on unven surfaces ${ }^{38)}$.

The data were analysed using the Statistical Package for Social Sciences (SPSS) version-20. The paired t-test was used to determine significant differences in the means of the outcome measures (HR, BP, 6-MWT, AV and FAC) within groups between baseline and week 8 , and the un-paired t-test was used to determine significant differences between the groups at baseline and week 8. A p-value of less than $0.05(\mathrm{p}<0.05)$ was considered significant.

\section{RESULTS}

This study had twenty participants: $12(60 \%)$ males and $8(40 \%)$ females. The mean age of the participants was 60.6 \pm 8.5 years. Their mean duration of stroke was $3.8 \pm 2.8$ months. Sixteen $(80 \%)$ and four $(20 \%)$ of the participants had ischaemic and haemorrhagic strokes, respectively. Ten (50\%) of the participants presented with left hemiparesis and the other half with right hemiparesis. Five (25\%) of the participants had a history of smoking and drinking alcohol, whereas $75 \%$ had no history of smoking and drinking alcohol. Sixteen (80\%) of the participants were married, $2(10 \%)$ were widows, and 2 $(10 \%)$ were single. Ten $(50 \%)$ of the participants were retirees, 7 (35\%) were working and $3(15 \%)$ were unemployed. The characteristics of the study participants are shown in Table 1.

In ErCG, the paired t-test indicated there were significant differences in the means of FAC, AV, 6-MWT, SBP and DBP from baseline to week $8(\mathrm{p}<0.03)$, but the mean value of HR had not changed significantly at week $8(\mathrm{p}=0.2408)($ Table 2$)$. 
Table 1. Characteristics of the study participants

\begin{tabular}{lcc}
\hline Characteristics & ErCG & \multicolumn{1}{c}{ CG } \\
\hline $\mathrm{N}(\%)$ & $10(50)$ & $10(50)$ \\
Age (Mean $\pm \mathrm{SD})$ (years) & $58.8 \pm 8.3$ & $62.4 \pm 8.8$ \\
$\begin{array}{l}\text { Duration of stroke } \\
\text { (Mean } \pm \mathrm{SD} \text { ) (months) }\end{array}$ & $3.5 \pm 2.6$ & $4.1 \pm 3.0$ \\
Type of stroke n (\%) & & \\
$\quad$ Ischaemic & $9(45)$ & $7(35)$ \\
$\quad$ Haemorrhagic & $2(10)$ & $2(10)$ \\
Side of stroke n (\%) & & \\
Left & $5(25)$ & $5(25)$ \\
Right & $5(25)$ & $5(25)$ \\
\hline
\end{tabular}

$\mathrm{N}$ : number of participants; \%: percentage; ErCG: ergometer cycling group; $\mathrm{CG}$ : control group; Mean $\pm \mathrm{SD}$ : mean plus or minus standard deviation
Table 2. Comparison of outcome measures between and within ErCG and CG at both baseline and week 8

\begin{tabular}{|c|c|c|}
\hline Outcome measure & ErCG $($ Mean \pm SD $)$ & $\mathrm{CG}($ Mean $\pm \mathrm{SD})$ \\
\hline \multicolumn{3}{|l|}{ FAC } \\
\hline Baseline & $2.8 \pm 0.6$ & $2.9 \pm 0.7$ \\
\hline Week 8 & $4.0 \pm 0.8^{\#}$ & $3.1 \pm 1.3$ \\
\hline \multicolumn{3}{|l|}{$\mathrm{AV}(\mathrm{m} / \mathrm{s})$} \\
\hline Baseline & $0.64 \pm 0.18^{*}$ & $0.46 \pm 0.22$ \\
\hline Week 8 & $0.74 \pm 0.18^{\# *}$ & $0.49 \pm 0.24$ \\
\hline \multicolumn{3}{|l|}{ 6-MWT (m) } \\
\hline Baseline & $278.7 \pm 58.06$ & $251.7 \pm 56.1$ \\
\hline Week 8 & $310.6 \pm 63.9^{\# *}$ & $268.5 \pm 55.1$ \\
\hline \multicolumn{3}{|l|}{ HR (bpm) } \\
\hline Baseline & $75.0 \pm 9.1$ & $81.2 \pm 4.0$ \\
\hline Week 8 & $72.3 \pm 6.3^{*}$ & $79.3 \pm 1.9$ \\
\hline \multicolumn{3}{|l|}{$\mathrm{SBP}(\mathrm{mmHg})$} \\
\hline Baseline & $133.9 \pm 12.2$ & $134.0 \pm 8.1$ \\
\hline Week 8 & $123.0 \pm 7.4^{\# *}$ & $133.1 \pm 8.3$ \\
\hline \multicolumn{3}{|l|}{ DBP $(\mathrm{mmHg})$} \\
\hline Baseline & $82.0 \pm 7.2$ & $83.6 \pm 7.5$ \\
\hline Week 8 & $73.2 \pm 9.0^{\# *}$ & $81.8 \pm 8.7$ \\
\hline
\end{tabular}

ErCG: ergometer cycling group; CG: control group; FAC: functional ambulatory category; AV: ambulatory velocity; 6-MWT: 6-Minute Walk Test; HR: heart rate; bpm: beats per minute; SBP: systolic blood pressure; DBP: diastolic blood pressure; mmHg: millimetres of Mercury; Mean \pm SD: mean plus or minus standard deviation

*significant differences in outcome measures between ErCG and CG at both baseline and week 8 . \#significant differences in outcome measures from baseline to week 8 in ErCG.

Likewise, there were no significant differences in the means of FAC, AV, 6-MWT, HR, SBP and DBP between baseline and week 8 ( $\mathrm{p}>0.05$ ) in CG (Table 2).

Analyses with the unpaired t-test indicated there were no significant differences in the means of FAC, AV, 6-MWT, HR, SBP and DBP between the ErCG and CG at baseline ( $p>0.05$ ) (Table 2). However, there were significant differences in the means of AV, 6-MWT, HR, SBP and DBP between the ErCG and CG at week 8 ( $\mathrm{p}<0.05)$ (Table 2).

\section{DISCUSSION}

This study investigated the effects of ergometer cycling on the ambulatory function and cardiovascular fitness of patients with stroke in the sub-acute phase. The findings of the study demonstrate that ergometer cycling in conjunction with conventional physiotherapy improved the ambulation and cardiovascular fitness of patients with stroke. The cardiovascular fitness of the participants improved more in ErCG than in CG as measured by resting heart rate and blood pressure. The HR and BP were significantly lower in ErCG than in CG at week 8. These results are in agreement with the results of previous studies $^{27,39,40)}$. Bateman et al. ${ }^{40)}$ reported that patients with stroke showed improved cardiovascular fitness after 12 weeks of cycle ergometer aerobic training.

The observed improvements in cardiovascular fitness may be related to improvements in sensorimotor function ${ }^{41}$. Other studies have shown that aerobic capacity or peak oxygen consumption and walking performance are reduced in elderly patients with stroke and that low oxygen consumption levels are associated with limited physical function when performing daily activities ${ }^{42}$. However, endurance exercise beneficially affects ambulatory blood pressure ${ }^{43)}$. Thus, increasing the fitness levels of patients with stroke may avert post-stroke problems including fatigue, increased energy cost of ambulation, falls and fractures, and poor quality of life $\mathrm{e}^{13)}$.

In this study, ambulatory velocity (AV) measured by the 10-MWT significantly increased in ErCG and also in comparison to CG at week 8. Similarly, the 6-MWT significantly increased in ErCG than in CG. These results support the findings of previous studies ${ }^{7,27,33)}$. Both ambulatory velocity and walking capacity in the experimental group showed greater improvement than in control group. The superior improvements in ambulatory velocity in ErCG may be attributable to the improved gait 
performance, strength and aerobic capacity of participants ${ }^{15,44)}$. The improvement in walking capacity may also be due to increased cardiovascular fitness and facilitation of motor learning ${ }^{44)}$. Furthermore, cycling exercise facilitates muscle control of the lower limbs, which may allow putting more weight on the affected leg while standing ${ }^{30)}$. Since the range of motion (ROM) in cycling is superior to that in walking, pedaling helps maintain the functional ROM of the lower limbs required to walk ${ }^{45}$. One advantage of cycling is that it involves the affected limb thereby facilitating use and limb control ${ }^{41)}$. Seki et al. observed significant increases in the muscle activities of the rectus femoris, tibialis anterior and soleus muscles in the affected leg of stroke patients during cycling compared with baseline isometric contraction ${ }^{46)}$. Thus, given the ambulatory velocity and walking capacity of patients in ErCG, they could safely perform community ambulation.

The FAC assesses the level of support or dependency a patient requires for ambulation ${ }^{44)}$. In this study, although FAC significantly increased in the ErCG, the mean difference was not statistically significant between the two groups at baseline and at week 8 (Tables 2). These findings are similar to observations of previous studies ${ }^{24}$, 27). Improvement in functional ambulatory ability in ErCG may have been due to improvements in the participants' aerobic capacity, strength and cardiopulmonary function as well as their increased $\mathrm{AV}$ of participants having been elicited by ergometer cycling ${ }^{15)}$. The FAC score of ErCG at the end of 8 weeks meant the participants could independently walk or ambulate freely especially, on level surfaces ${ }^{38)}$. Stationary cycling has been found useful for counteracting the consequences of immobility ${ }^{7}$. Katz-Leurer et al. reported that improvements in balance and motor abilities were greater in an experimental group (with less than a one-month history of stroke) than in a control group after three weeks of cycling exercises ${ }^{27}$. These findings suggest that early cycling exercise at the sub-acute phase may improve balance and motor performance ${ }^{15}$.

In conclusion, this study showed that ergometer cycling improved the ambulatory function and cardiovascular fitness of patients with stroke in the sub-acute phase. Therefore, ergometer cycling may be used to improve walking ability and cardiovascular health in stroke rehabilitation. However, due to the small sample size and the short duration of the intervention, further trials are needed to confirm or refute the effectiveness of ergometer cycling for the ambulatory function and cardiovascular fitness of patients with stroke in the sub-acute phase.

\section{Conflict of interest}

None.

\section{ACKNOWLEDGEMENT}

The authors thank the Research Open Access Publishing (ROAAP) Fund of the University of Illinois at Chicago for the financial support towards the open access publishing fee for this article.

\section{REFERENCES}

1) Go AS, Mozaffarian D, Roger VL, et al. American Heart Association Statistics Committee and Stroke Statistics Subcommittee: Heart disease and stroke statistics - 2014 update: a report from the American Heart Association. Circulation, 2014, 129: e28-e292. [Medline] [CrossRef]

2) Mazzocchio R, Meunier S, Ferrante S, et al.: Cycling, a tool for locomotor recovery after motor lesions? NeuroRehabilitation, 2008, 23: 67-80. [Medline]

3) Masiero S, Poli P, Rosati G, et al.: The value of robotic systems in stroke rehabilitation. Expert Rev Med Devices, 2014, 11: 187-198. [Medline] [CrossRef]

4) Sullivan BO, Schmitz TJ: Physical rehabilitation, 5th ed. Philadelphia: F.A. Davis Company, 2007, pp 725-727.

5) Da Cunha FT, Lim PA: Huma: a comparison of regular rehabilitation and regular rehabilitation with supported treadmill ambulation training for acute stroke patients. J Rehabil Res Dev, 2001, 38: 243-2452.

6) Carr JH, Shepherd RB: Stroke rehabilitation: guidelines for exercise and training to optimise motor skill. Oxford: Butterworth-Heinemann, 2003 , pp 209-233.

7) Kamps A, Schüle K: Cyclic movement training of the lower limb in stroke rehabilitation. Neurol Rehabil, 2005, 5: 1-12.

8) Ryan AS, Dobrovolny CL, Silver KH, et al.: Cardiovascular fitness after stroke: role of muscle mass and gait deficit severity. J Stroke Cerebrovasc Dis, 2000, 9: 185-191. [Medline] [CrossRef]

9) Lexell J, Dutta C: Sarcopenia and physical performance in old age: National Institute on Aging Workshop. Muscle Nerve Suppl, $1997,5: 5$.

10) Zun W, Lei W, Hongjuan F, et al.: Effect of low-intensity ergometer aerobic training on glucose tolerance in severely impaired nondiabetic stroke patients. J Stroke Cerebrovasc Dis, 2014, 23: 187-193. [CrossRef]

11) Smith NL, Barzilay JI, Shaffer D, et al.: Fasting and 2-hour postchallenge serum glucose measures and risk of incident cardiovascular events in the elderly: the Cardiovascular Health Study. Arch Intern Med, 2002, 162: 209-216. [Medline] [CrossRef]

12) Saunders DH, Greig CA, Young A, et al.: Physical fitness training for stroke patients. Cochrane Database Syst Rev, 2004, (1): CD003316 10.1002/14651858. CD003316.pub2. [Medline]

13) Kluding PM, Tseng BY, Billinger SA: Exercise and executive function in individuals with chronic stroke: a pilot study. J Neurol Phys Ther, 2011, 35: 11-17. [Medline] [CrossRef]

14) Dickstein R: Rehabilitation of gait speed after stroke: a critical review of intervention approaches. Neurorehabil Neural Repair, 2008, 22: 649-660. [Medline] [CrossRef]

15) Barbosa D, Santos CP, Martins M: The application of cycling and cycling combined with feedback in the rehabilitation of stroke patients: a review. J Stroke Cerebrovasc Dis, 2015, 24: 253-273. [Medline] [CrossRef] 
16) Frimpong E, Olawale OA, Antwi DA, et al.: Task- oriented circuit training improves ambulatory functions in acute stroke: a randomized controlled trial. J. Med. Med. Sci, 2014, 5: 169-175.

17) Globas C, Becker C, Cerny J, et al.: Chronic stroke survivors benefit from high-intensity aerobic treadmill exercise: a randomized control trial. Neurorehabil Neural Repair, 2012, 26: 85-95. [Medline] [CrossRef]

18) van de Port IG, Wevers LE, Lindeman E, et al.: Effects of circuit training as alternative to usual physiotherapy after stroke: randomised controlled trial. BMJ, 2012, 344: e2672. [Medline] [CrossRef]

19) Toledano-Zarhi A, Tanne D, Carmeli E, et al.: Feasibility, safety and efficacy of an early aerobic rehabilitation program for patients after minor ischemic stroke: a pilot randomized controlled trial. NeuroRehabilitation, 2011, 28: 85-90. [Medline]

20) Cooke EV, Tallis RC, Clark A, et al.: Efficacy of functional strength training on restoration of lower-limb motor function early after stroke: phase I randomized controlled trial. Neurorehabil Neural Repair, 2010, 24: 88-96. [Medline] [CrossRef]

21) Moore JL, Roth EJ, Killian C, et al.: Locomotor training improves daily stepping activity and gait efficiency in individuals poststroke who have reached a "plateau" in recovery. Stroke, 2010, 41: 129-135. [Medline] [CrossRef]

22) Smith PS, Thompson M: Treadmill training post stroke: are there any secondary benefits? A pilot study. Clin Rehabil, 2008, 22: 997-1002. [Medline] [CrossRef]

23) Eich HJ, Mach H, Werner C, et al.: Aerobic treadmill plus Bobath walking training improves walking in subacute stroke: a randomized controlled trial. Clin Rehabil, 2004, 18: 640-651. [Medline] [CrossRef]

24) Pohl M, Mehrholz J, Ritschel C, et al.: Speed-dependent treadmill training in ambulatory hemiparetic stroke patients: a randomized controlled trial. Stroke, 2002, 33: 553-558. [Medline] [CrossRef]

25) Ada L, Dean CM, Lindley R: Randomized trial of treadmill training to improve walking in community-dwelling people after stroke: the AMBULATE trial. Int J Stroke, 2013, 8: 436-444. [Medline] [CrossRef]

26) Kang HK, Kim Y, Chung Y, et al.: Effects of treadmill training with optic flow on balance and gait in individuals following stroke: randomized controlled trials. Clin Rehabil, 2012, 26: 246-255. [Medline] [CrossRef]

27) Katz-Leurer M, Sender I, Keren O, et al.: The influence of early cycling training on balance in stroke patients at the subacute stage. Results of a preliminary trial. Clin Rehabil, 2006, 20: 398-405. [Medline] [CrossRef]

28) Raasch CC, Zajac FE: Locomotor strategy for pedaling: muscle groups and biomechanical functions. J Neurophysiol, 1999, 82: 515-525. [Medline]

29) Brown DA, Kautz SA, Dairaghi CA: Muscle activity adapts to anti-gravity posture during pedalling in persons with post-stroke hemiplegia. Brain, 1997, 120: 825-837. [Medline] [CrossRef]

30) Kim SJ, Cho HY, Kim YL, et al.: Effects of stationary cycling exercise on the balance and gait abilities of chronic stroke patients. J Phys Ther Sci, 2015, 27: 3529-3531. [Medline] [CrossRef]

31) Sibley KM, Tang A, Brooks D, et al.: Feasibility of adapted aerobic cycle ergometry tasks to encourage paretic limb use after stroke: a case series. J Neurol Phys Ther, 2008, 32: 80-87. [Medline] [CrossRef]

32) Ferrante S, Ambrosini E, Ravelli P, et al.: A biofeedback cycling training to improve locomotion: a case series study based on gait pattern classification of 153 chronic stroke patients. J Neuroeng Rehabil, 2011, 8: 47. [Medline] [CrossRef]

33) Holt R, Kendrick C, McGlashan K, et al.: Static bicycle training for functional mobility in chronic stroke. Physiotherapy, 2001, 87: 257-260. [CrossRef]

34) Pacheco L, Oglivie R, Chong S, et al.: Arm and leg cycling with functional electrical stimulation improves walking after incomplete spinal cord injury. Neuroscience, 2011, 2: 1-11.

35) Borg GA: Psychophysical bases of perceived exertion. Med Sci Sports Exerc, 1982, 14: 377-381. [Medline] [CrossRef]

36) Lord SE, Rochester L: Measurement of community ambulation after stroke: current status and future developments. Stroke, 2005, 36: 1457-1461. [Medline] [CrossRef]

37) Dean CM, Richards CL, Malouin F: Task-related circuit training improves performance of locomotor tasks in chronic stroke: a randomized, controlled pilot trial. Arch Phys Med Rehabil, 2000, 81: 409-417. [Medline] [CrossRef]

38) Holden MK, Gill KM, Magliozzi MR, et al.: Clinical gait assessment in the neurologically impaired. Reliability and meaningfulness. Phys Ther, 1984, 64: 35-40. [Medline]

39) Lennon O, Carey A, Gaffney N, et al.: A pilot randomized controlled trial to evaluate the benefit of the cardiac rehabilitation paradigm for the non-acute ischaemic stroke population. Clin Rehabil, 2008, 22: 125-133. [Medline] [CrossRef]

40) Bateman A, Culpan FJ, Pickering AD, et al.: The effect of aerobic training on rehabilitation outcomes after recent severe brain injury: a randomized controlled evaluation. Arch Phys Med Rehabil, 2001, 82: 174-182. [Medline] [CrossRef]

41) Potempa K, Lopez M, Braun LT, et al.: Physiological outcomes of aerobic exercise training in hemiparetic stroke patients. Stroke, 1995, 26: 101-105. [Medline] [CrossRef]

42) Billinger SA, Taylor JM, Quaney BM: Cardiopulmonary response to exercise testing in people with chronic stroke: a retrospective study. Stroke Res Treat, 2012, 2012: 987637. [Medline]

43) Cornelissen VA, Buys R, Smart NA: Endurance exercise beneficially affects ambulatory blood pressure: a systematic review and meta-analysis. J Hypertens, 2013, 31: 639-648. [Medline] [CrossRef]

44) Saunders DH, Greig CA, Mead GE, et al.: Physical fitness training for stroke patients. Cochrane Database Syst Rev, 2009, (4): CD003316 10.1002/14651858. CD003316.pub3. [Medline]

45) Lin SI, Lo CC, Lin PY, et al.: Biomechanical assessments of the effect of visual feedback on cycling for patients with stroke. J Electromyogr Kinesiol, 2012, 22: 582-588. [Medline] [CrossRef]

46) Seki K, Sato M, Handa Y: Increase of muscle activities in hemiplegic lower extremity during driving a cycling wheelchair. Tohoku J Exp Med, 2009, 219: 129-138. [Medline] [CrossRef] 